\title{
Absence of evidence or methodological issues? \\ Commentary on "Stay-at-home policy is a case of exception fallacy: an internet-based ecological study"
}

\author{
Gideon Meyerowitz-Katz* Lonni Besançon ${ }^{\dagger}$ \\ Antoine Flahault ${ }^{\ddagger} \quad$ Raphael Wimmer ${ }^{\S}$
}

March 16, 2021

We read with interest the paper written by Savaris et al. entitled "Stay-at-home policy is a case of exception fallacy: an internet-based ecological study" [4]. We believe that the topic of whether non-pharmaceutical interventions (NPIs) have an impact on COVID-19 mortality is a key metric that is important to measure, and applaud the authors for attempting to do so. However, we believe that several key deficiencies within the methodology make the conclusions - that the authors found no evidence that COVID-19 deaths were reduced by staying at home - largely meaningless. These are presented below:

1. The use of Google mobility data is convenient, but carries with it significant disadvantages. Measures within this data are inherently opaque, i.e. there is no information on how mobility is calculated, which individuals make up the sample, or which biases that this data may have. Without such information, it is impossible to assess whether, for example, the "residential" category is a reasonable measure of people staying at home, or whether it is unrelated. While such mobility data is not unreasonable, it carries with it disadvantages that may have influenced the results.

*Faculty of Health and Society, University of Wollongong, Wollongong, Australia, gidmk.healthnerd@gmail.com

'Faculty of Information Technology, Monash University, Clayton, Australia, lonni.besancon@gmail.com

¿University of Geneva, Geneva, Switzerland, Antoine.Flahault@unige.ch

$\S$ University of Regensburg, Regensburg, Germany, raphael.wimmer@ur.de 
2. The authors justify the use of such data by citing two prior COVID-19 projects that also relied on it $[1,5$. However, unlike prior works, the authors used only the "residential" category of the mobility data. In comparison, Delen et al. [1] combined data from Google and Apple and considered the overall mobility of users instead of focusing solely on residential places. Similarly Vokó and Pitter [5] considered overall patterns of mobility over workplaces, retails, grocery, parks, etc, instead of only changes over residential places. Focusing on residential changes, as noted by Google [2], is a major error. As people already spend most of their time at home, this measure is the least likely to show any impact when behaviour changes. Google mobility data has no raw information on movement and only looks at proportional changes in an opaque mobility score - thus, using only the metric which is least likely to show any impact will lead to a bias towards the null hypothesis.

3. Ecological studies of this kind are also geared towards null findings. As one example, the country of Australia is included as a unit of analysis. However, during the time period of the study, Australia had a unique situation, with a single state (Victoria) locking down for months while the rest of the country opened up. At the country level this difference becomes almost negligible, with an increase of $<10 \%$ across Australia in "residential" mobility. However, in Victoria alone, the increase exceeded $20 \%$ for this entire period. Any comparisons to Australia as a whole during the Victorian lockdown are hence unreliable as a measure of the key metrics of interest, and while this is perhaps most significant for Australia it is clearly not limited to a single nation. This limitation is noted in the discussion, but it is not just a notation but a fundamental limitation that makes the final numbers largely arbitrary.

4. Including only countries with more than 100 overall COVID-19 deaths in the analysis excludes countries such as New Zealand, Singapore, and Vietnam that had success with rapid, strict measures. This introduces a bias towards countries without effective measures and therefore increases the chance that no difference between countries can be found.

5. The analysis also is intrinsically flawed, as it involves regressing two collinear values. COVID-19 deaths and "residential" behaviour, insofar as that may be a marker of people staying at home, are directly related as rising deaths cause both government restrictions and changes in behaviour.

6. The analysis fails to take into account any time lags between rising death rates and changes in behaviour. This is problematic for numerous reasons, in particular the fact that reported deaths lag several weeks behind actual deaths, due to the fact that reporting is not immediate in any country in the world. The analysis should instead take into account the fact that the impact of a specific NPI can only be seen after a delay of two or three weeks on mortality (see e.g., [3]). 
7. The chosen statistical approach may not be able to detect an effect of staying at home (or changes in mobility) on mortality at all. The authors did not demonstrate that their approach would actually be able to detect an effect if it existed.

Thankfully, the authors published source code and data that allow for replicating their analysis. We could reproduce the results shown in Fig. 2 of their paper using the provided dataset and the Jupyter notebook. However, it seems that the p-values in this figure have not been corrected for multiple comparisons, so the legend is misleading. To determine whether the approach could actually detect an effect of lockdowns, we constructed a data set with five hypothetical countries where changes in mobility (or the lack thereof) had an obvious effect on mortality (Figure 1). When running this dataset through the Jupyter notebook, none of the comparisons were statistically significant at $\mathrm{p}<.05$ (Figure 2). According to the authors' criteria, this would mean that even for our handcrafted dataset, no effect of lockdowns could be found.

Running a dataset with random data (Figure 3 ) through the notebook resulted in one of ten comparisons being statistically significant at $\mathrm{p}$ $<.05$ (Figure 4). After asking the lead author for a dataset that could demonstrate the effect of lockdowns, he sent a dataset with two countries for which the notebook indeed outputs a p-value of $<.0001$ (Figure 5 . top). However, just adding a little noise to the dataset resulted in a nonsignificant result again (Figure 5 bottom). The source code and datasets can be found on our GitHub repository 6 .

This indicates that the proposed method a) cannot reliably detect an effect of lockdowns even if it should exist, b) cannot distinguish meaningful data from random values, and c) is susceptible to noise in the data. Overall, this seems to make the approach unsuitable for detecting even obvious effects of a lockdown in artificial or real-life data.

While the question of whether NPIs can influence COVID-19 deaths is of great importance, the issues we have highlighted seriously hinder the conclusions made by the authors. That they found no statistically significant differences between regions on these metrics may simply be a function of their chosen methodology and the inherent limitations of the mobility dataset, and has little to do with the matter at hand. It appears likely that the methodology could not detect an effect of staying at home on mortality even if one were to exist. 


\section{References}

[1] Dursun Delen, Enes Eryarsoy, and Behrooz Davazdahemami. "No Place Like Home: Cross-National Data Analysis of the Efficacy of Social Distancing During the COVID-19 Pandemic". EN. In: JMIR Public Health and Surveillance 6.2 (May 2020), e19862. DOI: 10.2196/19862.

[2] Google. Understand the Data - Community Mobility Reports Help. https : // support . google . com/covid19-mobility/answer/9825414? hl=en (Visited on 03/15/2021).

[3] You Li et al. "The Temporal Association of Introducing and Lifting NonPharmaceutical Interventions with the Time-Varying Reproduction Number (R) of SARS-CoV-2: A Modelling Study across 131 Countries". en. In: The Lancet Infectious Diseases 21.2 (Feb. 2021), pp. 193-202. ISSN: 14733099. DOI: 10.1016/S1473-3099(20)30785-4.

[4] R. F. Savaris et al. "Stay-at-Home Policy Is a Case of Exception Fallacy: An Internet-Based Ecological Study". en. In: Scientific Reports 11.1 (Dec. 2021), p. 5313. ISSN: 2045-2322. DOI: 10.1038/s41598-021-84092-1.

[5] Zoltán Vokó and János György Pitter. "The Effect of Social Distance Measures on COVID-19 Epidemics in Europe: An Interrupted Time Series Analysis". en. In: GeroScience 42.4 (Aug. 2020), pp. 1075-1082. ISSN: 2509-2723. DOI: $10.1007 / \mathrm{s} 11357-020-00205-0$.

[6] Raphael Wimmer. RaphaelWimmer/COVID-19-Mobility-vs-Mortality. https://github.com/RaphaelWimmer/COVID-19-Mobility-vs-Mortality Mar. 2021. (Visited on 03/15/2021). 
Figure 1: Five hypothetical countries where deaths (orange) are lower if more people stay at home (blue).
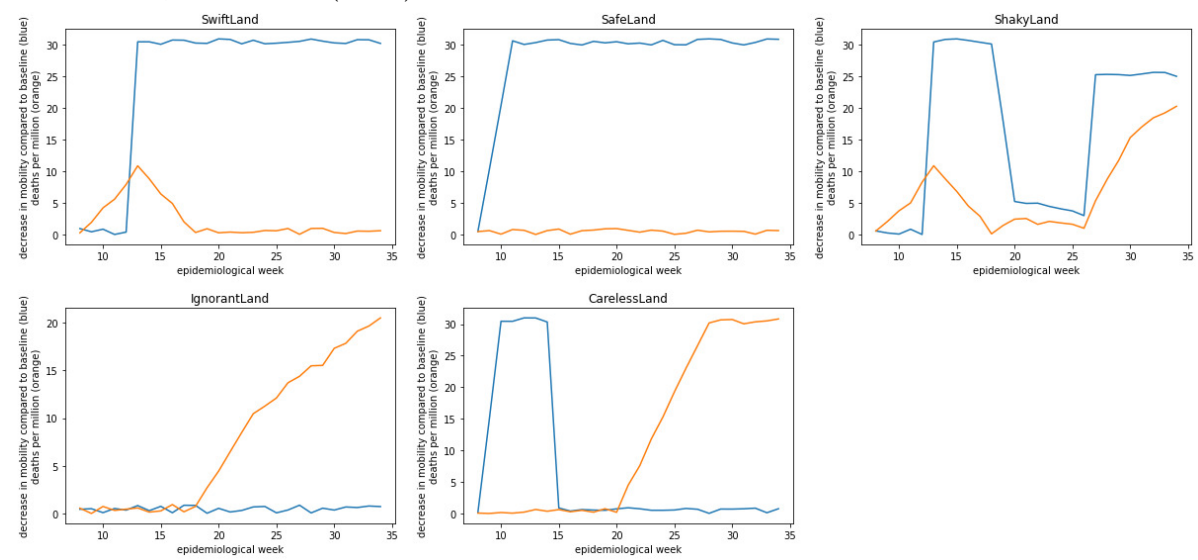
Figure 2: Running the dataset from Figure 1 through the authors' Jupyter notebook results in no statistically significant correlations, i.e. according to the authors' criteria, this dataset would not demonstrate an effect of lower mobility on mortality.

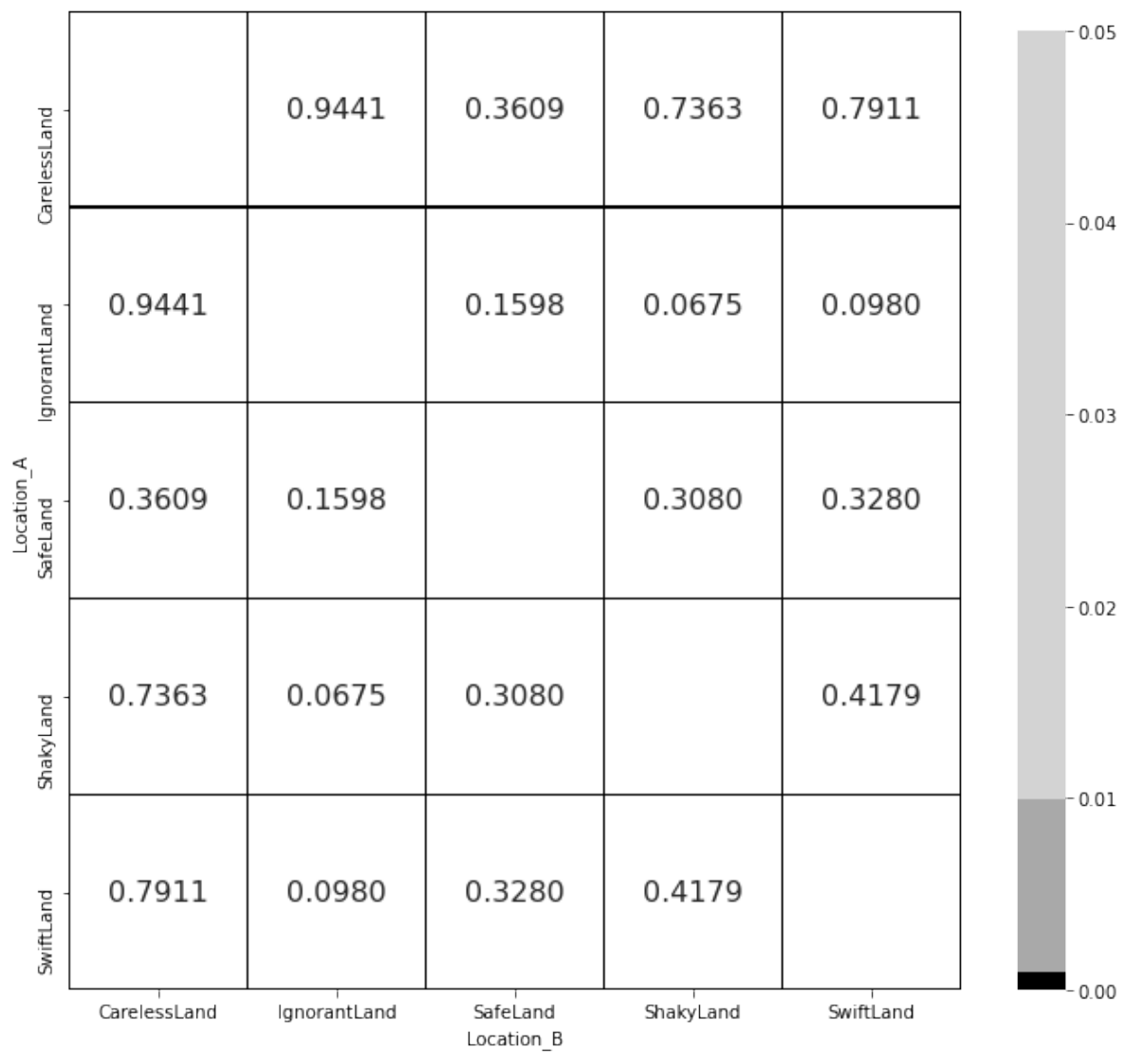


Figure 3: Five datasets with random time series.
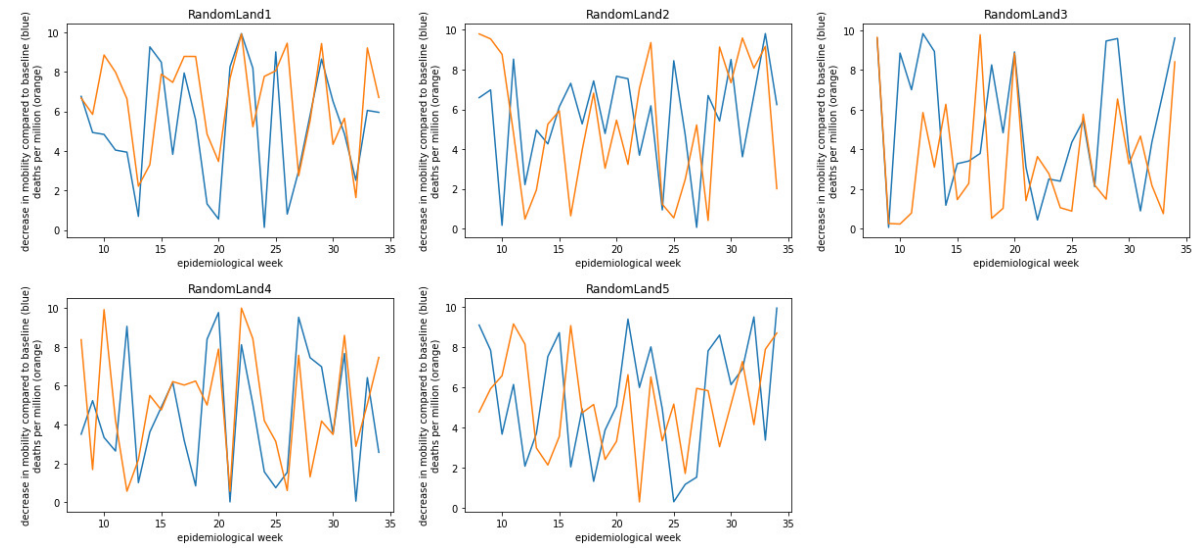
Figure 4: Running the random dataset from Fig. 3 through the authors' Jupyter notebook results in one statistically significant correlation $(=10 \%$ of comparisons).

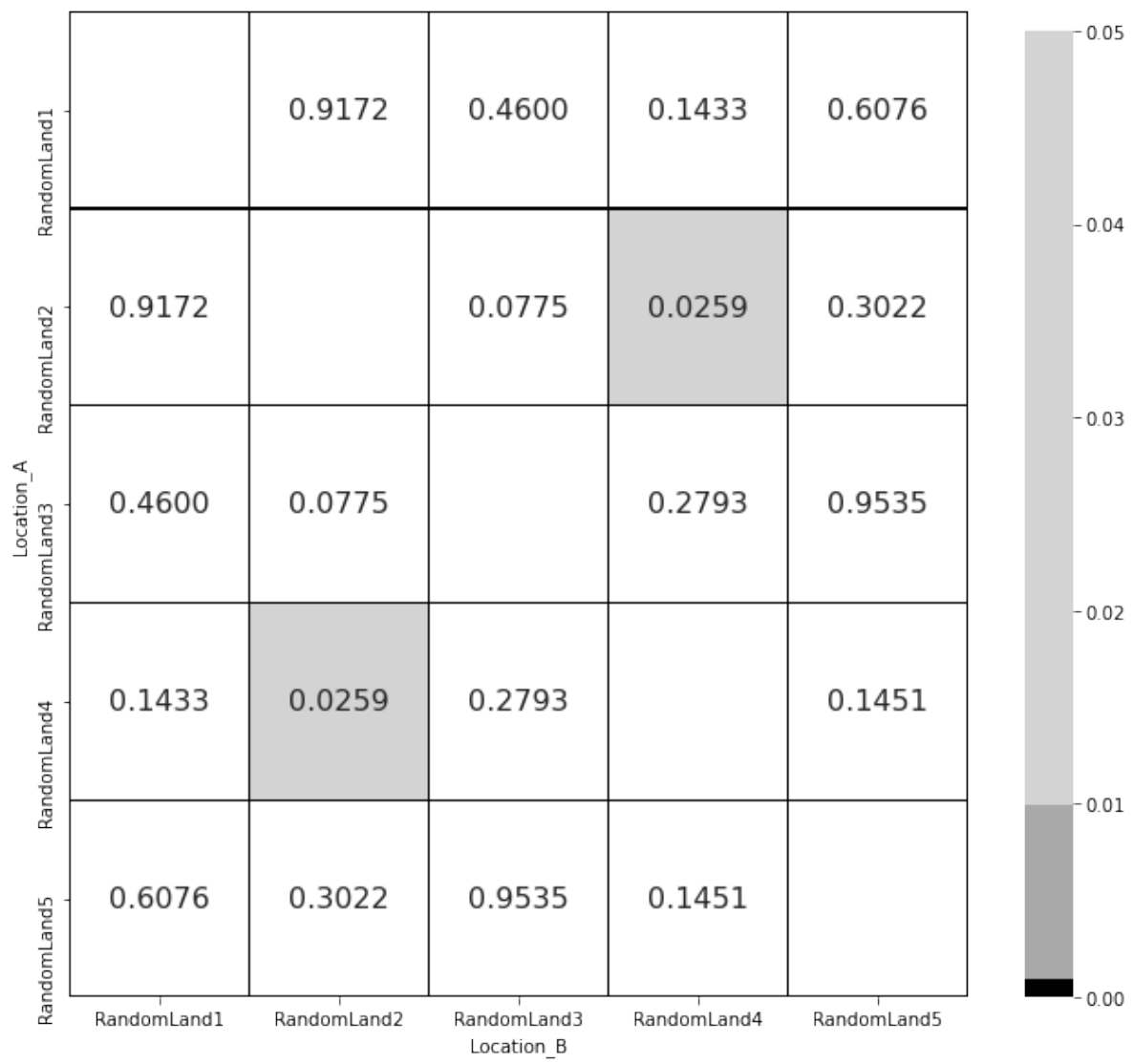


Figure 5: A handcrafted dataset by R.F. Savaris (top, personal communication) indeed results in a statistically significant correlation $(\mathrm{p}<.0001)$. However, adding noise to the dataset quickly destroys statistical significance (bottom).
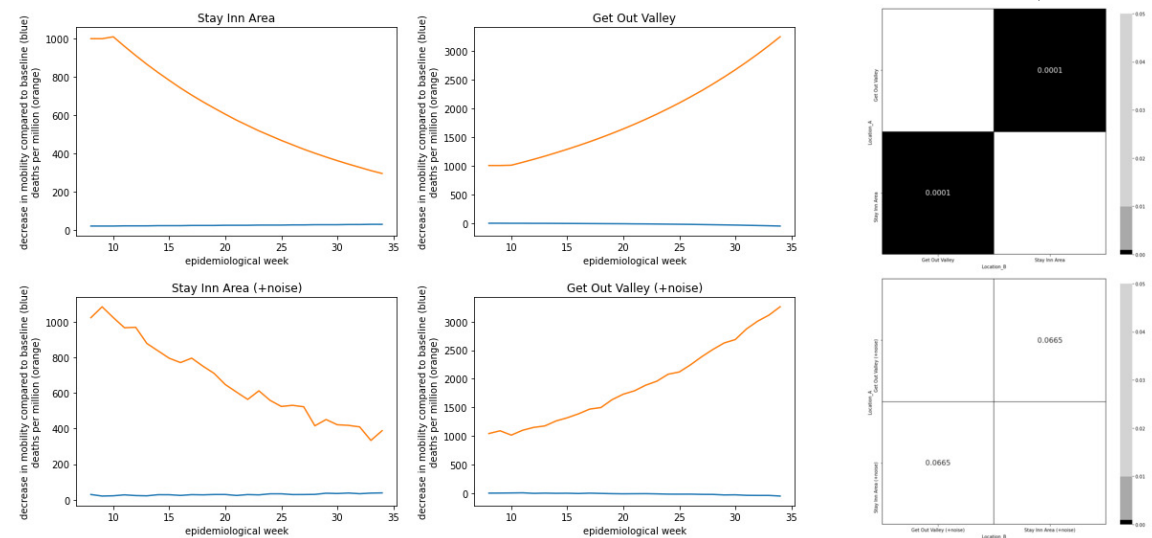